\title{
Transatlantica
}

Revue d'études américaines. American Studies Journal

\section{André Kaenel, Catherine Lejeune, Marie-Jeanne Rossignol. Cultural Studies. Etudes Culturelles.}

Nancy : Presses Universitaires de Nancy, 2003, 193 p.

\section{Pierre Guerlain}

\section{OpenEdition}

\section{Journals}

Édition électronique

URL : http://journals.openedition.org/transatlantica/844

DOI : $10.4000 /$ transatlantica.844

ISSN : 1765-2766

Éditeur

AFEA

Référence électronique

Pierre Guerlain, «André Kaenel, Catherine Lejeune, Marie-Jeanne Rossignol. Cultural Studies. Etudes Culturelles. », Transatlantica [En ligne], 1 | 2005, mis en ligne le 22 avril 2006, consulté le 29 avril 2021. URL : http://journals.openedition.org/transatlantica/844 ; DOI : https://doi.org/10.4000/transatlantica. 844

Ce document a été généré automatiquement le 29 avril 2021.

\section{(c)}

Transatlantica - Revue d'études américaines est mis à disposition selon les termes de la licence Creative Commons Attribution - Pas d'Utilisation Commerciale - Pas de Modification 4.0 International. 


\section{André Kaenel, Catherine Lejeune, Marie-Jeanne Rossignol. Cultural Studies. Etudes Culturelles.}

Nancy : Presses Universitaires de Nancy, 2003, 193 p.

\section{Pierre Guerlain}

1 Le titre de cet ouvrage et le choix des polices, respecté ici, permettent d'entrer dans le vif du sujet selon un angle d'attaque sémantique. Les auteurs sentent bien que la traduction française de "Cultural Studies» pose problème et que les «études culturelles » en France n'ont pas la même connotation que les «Cultural Studies » ou "Cultstuds", dans les pays anglophones. Les auteurs pratiquent dès le titre ce qui caractérise les Cultural Studies (CS par la suite), à savoir une transgression typographique, ils passent du gras à l'italique et anglicisent la norme française habituelle dans les titres car ils écrivent "Culturelles» en gardant la majuscule habituelle en anglais mais non en français (transgression non reprise dès la page de garde). Intentionnelle ou inconsciente, cette transgression fait l'effet d'un anglicisme, comme si les Cultural Studies avaient du mal à passer la barrière de la langue ou des habitudes culturelles françaises, ce qui interpelle tant la « French Theory » a contribué à transformer les CS aux Etats-Unis.

2 C'est précisément ce passage difficile que l'ouvrage présente, interroge et problématise. Ce travail est organisé en trois parties : 1) L'émergence des CS de Birmingham à New York : un double engagement politique et théorique, 2) Les CS face aux disciplines, en sciences sociales et humaines, 3) Regards sur la littérature : nouveaux textes, nouvelles lectures. Ce découpage est le résultat d'une adaptation à une scène intellectuelle ou à un public français, puisqu'il réintroduit un découpage entre histoire, sciences sociales et littérature alors que les CS ont précisément rendu flou, voire ont, dans certaines universités, aboli ces clivages. Certains textes sont rédigés en français, d'autres en anglais. Les deux articles d'André Kaenel, très présent dans cet ouvrage, sont écrits dans un bel anglais qui est lui-même typique du style CS américain récent. Les deux contributions de Kaenel, placées dans deux parties différentes, mais à la suite l'une de 
l'autre interrogent la division entre civilisation et littérature dans nos universités. Division d'origine administrative qui tend à être acceptée sur le plan scientifique, comme si elle allait de soi, idée que les CS précisément « déconstruisent ».

3 Je propose ici de présenter l'ouvrage en faisant quelques remarques de commentaire, puis, dans un second temps, d'en faire une analyse interprétative qui débordera largement sur une question plus vaste : que faire des CS ?

I.

4 L'ouvrage fait suite à un colloque fort stimulant qui s'était tenu à l'Institut d'Anglais Charles V (Université Paris 7) mais ne reprend qu'une partie des communications, et ceci pas seulement pour des raisons de place puisque tous les éléments critiques des CS ont disparu du volume qui fait suite au colloque. Le choix des textes, qui présentent tous un intérêt, correspond probablement à un désir de dissémination des CS en France mais mutile la réflexion globale en ne donnant pas assez la parole à la critique. Comme toute discipline ou "champ disciplinaire », les CS peuvent s'enrichir du débat avec leurs critiques internes ou externes. On peut donc regretter le choix partisan effectué par les trois directeurs.

5 Le premier article, «Stuart Hall, Cultural Studies and the British Left » que l'on doit à Jeremy Tranmer est essentiel pour comprendre l'histoire des CS. Tranmer retrace bien l'inscription des CS dans le marxisme britannique et les défis et remises en cause des formes dominantes de ce marxisme dans les années 50 et 60 . Il fait le lien entre le contexte politique et le discours des théoriciens des CS très fortement influencé par Gramsci. Au-delà de son intérêt historique, l'article de Jeremy Tranmer permet aussi de mesurer la distance parcourue entre la Grande-Bretagne des années 50 et les Etats-Unis des années 90. L'analyse critique des Britanniques semble parfois s'être diluée dans une célébration des catégories du marché, et les marxistes d'antan sont remplacés par des penseurs qui n'évoquent plus les conditions de production de la culture mais s'attachent, de façon typiquement postmoderne, à la surface des objets, textes, films ou chansons. Les CS ont bousculé d'autres disciplines mais elles ont elles-mêmes une histoire faite des renversements et adaptation qu'il serait utile d'étudier.

6 Guillaume Marche offre, si l'on peut dire, une étude de cas dans son article sur la "théorie queer et la dynamique du mouvement gai et lesbien». Etude de cas car la théorie queer est une province des CS et Marche s'attache à montrer le lien entre texte et société, qu'il problématise. J'ai particulièrement apprécié la section qu'il intitule « la théorie queer, critique du simulacre identitaire ». Il y a là l'ébauche d'une critique légitime des effets d'un multiculturalisme de la différence et de la prédilection pour le concept de communauté dans les sciences humaines et la pensée journalistique américaine. Marche rejette le modèle ethnique souvent utilisé dans l'analyse du mouvement gai et lesbien, modèle induit, je pense, par l'évolution des CS qui a quitté le discours de classes sociales pour celui de la diversité communautaire.

7 Stephan Herbrechter aborde dans son article, "The translation of CS ", le thème mis en avant en introduction ici. Il note l'hésitation grammaticale pour évoquer, en anglais les CS. (What is (are?) Cultural Studies?), hésitation grammaticale qui renvoie au statut. Le singulier s'impose s'il s'agit d'une discipline, le pluriel s'il s'agit d'un regroupement de méthodologies diverses appliquées à divers objets. Herbrechter offre quelques pistes pour analyser le passage transatlantique des CS d'Angleterre aux Etats-Unis et voit dans la « civilisation » une retraduction française des CS (il propose aussi Landeskunde pour l'allemand, ce qui pose encore plus de problèmes). Les retraductions non-anglophones 
de ce que Herbrechter appelle "the Anglo-American CS paradigm " ne sont pas que linguistiques mais aussi culturelles. De façon intéressante, il parle des CS comme d'un marché qui a connu un «boom » et qui s'essouffle. La question qui se pose donc ici est : les CS ne seraient-elles qu'une mode intellectuelle, bientôt chassée par une autre ? Si les CS sont une mode comment les rapprocher de Clio, multiséculaire, ou de la sociologie, discipline établie où les phénomènes de mode existent mais dans le champ de son débat interne?

8 Marie-Jeanne Rossignol lance la deuxième partie avec brio. Son article « Histoires culturelles : l'histoire et les CS, en France et aux Etats-Unis » appréhende deux types de relations, celles entre les CS et l'histoire d'une part, et les différences de contextes de réception des CS entre la France et les Etats-Unis, d'autre part. Elle note que les CS n'ont pas beaucoup influencé les historiens français qui leur préfèrent l'histoire culturelle. Cependant, certains théoriciens prisés des tenants des CS, Foucault, par exemple, ont fait bouger la réflexion en histoire. Rossignol s'intéresse plus à l'impact des CS et du postmodernisme sur l'histoire et à la résistance des historiens qu'aux CS en elles-mêmes et elle fait dialoguer Hayden White et Roger Chartier qui s'avère un défenseur de l'histoire-« discours de vérité » particulièrement convaincant. L'histoire culturelle apparaît comme une réponse aux CS, réponse qui prend en compte certaines mises en cause mais rejette le linguistic turn comme le cultural turn dans lesquels les discours peuvent ne plus avoir de référents dans le réel. C'est l'histoire et ses évolutions qui intéressent Marie-Jeanne Rossignol et elle dresse un tableau des évolutions tant aux Etats-Unis qu'en France qui est particulièrement intéressant. Si elle note bien les apports possibles et la fertilisation croisée des CS et de l'histoire, elle ne creuse pas la critique profonde des CS telles qu'elles sont parfois critiquées aux Etats-Unis. La remarque de Chartier qu'elle cite est très pertinente : là où l'historien ne peut abolir le réel même s'il ou elle ne peut que l'approcher, un partisan de Hayden White, ou un Baudrillard, risque de le fantasmer. Dans leur passage transatlantique les CS, qui sont entre temps devenues postmodernes sous l'influence de penseurs français comme Derrida, ont parfois évolué vers des jeux de discours, voire des jeux de mots, portant sur des objets de la culture de masse, appréhendés de façon délibérément superficielle. Marie-Jeanne Rossignol est la seule à insister sur cet apport de penseurs français, d'ailleurs fort divers, et que le regroupement sous le terme de "French theory" constitue en groupe plutôt problématique car c'est la nationalité qui est retenue par la classification plutôt que la théorie elle-même.

Catherine Lejeune fait ensuite un travail semblable à celui de Rossignol en ce sens qu'elle étudie l'impact des CS sur l'anthropologie et se demande si cette discipline serait menacée. Là, cependant, les enjeux sont différents car les CS se placent sur un terrain plus proche de l'anthropologie. On pourra noter ici que c'est aussi vrai de la sociologie, à tel point que nombre d'ouvrages qui en France seraient classés dans les rayons de la sociologie, le sont en CS dans les pays anglophones. Lejeune présente et analyse la lutte entre CS et anthropologie, lutte dont les premières hostilités sont le fait des CS. Cette lutte pose le problème des frontières disciplinaires et Lejeune aborde là un problème important que l'émergence des CS ne fait que souligner. Elle montre bien que, comme toutes les frontières, celle entre CS et anthropologie, est poreuse et que certains ont peur d'une invasion dans les départements universitaires. Une discipline est, en effet, un champ intellectuel, mais aussi, et peut-être surtout, une division administrative à l'université où les enjeux de postes et de crédits dans un environnement économique défavorable sont cruciaux. Il est parfois assez difficile de 
démêler les enjeux intellectuels des enjeux administratifs et financiers, qui sont évidemment différents aux Etats-Unis de ce qu'ils sont en France. C'est d'ailleurs l'une des raisons qui expliquent pourquoi les débats ne sont pas engagés de la même façon. Catherine Lejeune évoque vers la fin de son article les Subaltern Studies qu'elle définit ainsi : «domaine apparenté aux CS, à l'intersection du post-modernisme et du postcolonialisme ", créé par des intellectuels indiens souvent exilés. Il y a là une autre piste à explorer : la valse des étiquettes disciplinaires. Si les CS sont distinctes des Subaltern Studies et du post-colonialisme on peut se poser la question de leur spécificité. Chaque groupe invente-t-il sa discipline, les Subaltern Studies, sont-elles les CS pour universitaires indiens en exil? Nous verrons plus loin les difficultés posées par les logiques identitaires qui se traduisent ou tentent de se traduire par l'invention d'une discipline.

Geneviève Sellier s'attache ensuite à l'étude de «l'apport des gender studies aux études filmiques ", gender studies qui sont assimilées à ou englobées par les CS mais l'auteur(e) traite pourtant les CS de façon différente de ses co-auteurs, semble-t-il. Elle écrit ainsi que les gender studies « ont droit de cité, chez nos voisins d'Outre Manche et d'Outre Atlantique dans tous les champs disciplinaires ", alors que d'autres voient dans les CS une discipline ou un champ disciplinaire à part entière. Comme les deux auteures précédentes, elle s'intéresse surtout à un champ disciplinaire, ici le cinéma, après l'histoire et l'anthropologie, et à l'impact des gender studies sur l'étude du cinéma. On aurait pu aussi parler de féminisme ou de women's studies. Le féminisme américain a en effet eu un impact considérable sur la façon d'envisager la culture, la politique et l'enseignement supérieur et a, entre autres, rendu visibles des personnes et des problématiques invisibilisées jusque dans les années 60 . Mais peut-on affirmer que ce sont les CS qui posent «le problème de l'inscription sociale des productions culturelles" ? Ici se pose le problème évoqué plus haut dans le choix des textes. Cet article, tout à fait intéressant par ailleurs, n'échappe pas au côté propagandiste des CS qui caractérise l'ouvrage. La phrase citée précédemment suppose que l'histoire et la sociologie, voire même la philosophie, n'avaient pas déjà posé le problème de l'inscription sociale des productions culturelles, comme si Adorno et l'Ecole de Francfort n'avaient jamais réfléchi à ce problème, comme si des années de marxisme en tout genre n'avaient pas fait de ce problème le centre leur leurs préoccupations, d'ailleurs de façon souvent problématique puisque l'œuvre risquait de disparaître derrière la réflexion sur l'inscription sociale. Les CS n'ont pas réinventé toute la sociologie et ce qui est présenté comme un apport novateur n'est ici qu'une poursuite d'un travail antérieur.

11 Il est clair que les CS correspondent à un phénomène de mode culturelle et comme pour toutes les modes, ses partisans affirment sa nouveauté radicale. Mais il s'agit là d'un effet d'annonce dont on peut aisément montrer l'inexactitude. Comme toutes les modes et mouvements soumis au système de la mode, les CS se fragmentent et ses diverses factions ou fractions sont en lutte pour la notoriété. Certains inspirateurs ou instigateurs, comme Frank Kermode ou Frank Lentricchia, sont revenus de leurs amours initiales et tiennent des discours semblables à ceux des "traditionalistes" auxquels ils s'opposaient. Ceci ne veut pas dire que les CS soient dénuées d'intérêt, bien sûr. Sellier termine son article par un plaidoyer: "Mieux faire connaître ces recherches en France ", dans lequel elle affirme que «les études filmiques en France résistent des quatre fers à la contamination anglo-américaine des gender studies ». Il me semble que c'est quelque peu calomnier les études filmiques en France (mais je ne 
suis pas spécialiste) et que le mot ce « contamination » fait glisser l'argumentation sur un terrain douteux car l'on sait bien qu'il fait partie de l'argumentaire xénophobe et raciste traditionnel.

André Kaenel signe les deux articles suivants, écrits en anglais et qui, contrairement aux articles rédigés en français dans ce recueil, sont précédés d'un résumé en français. Il aurait été souhaitable qu'un résumé en anglais figure au début des articles rédigés en français. Le premier de ces articles renvoie à un thème qu'André Kaenel a souvent abordé avec bonheur, celui de l'histoire des American Studies aussi bien aux Etats-Unis qu'en Europe. Comme Rossignol et Lejeune, Kaenel analyse donc l'impact des CS sur un champ disciplinaire, celui des American Studies et il faut une étude comparative entre les Etats-Unis et l'Europe. Kaenel fait une bonne analyse des débats autour du nom même des American Studies aux Etats-Unis et présente, à la suite d'Aronowitz, les CS comme l'instrument du changement dans ce champ disciplinaire. Concernant l'Europe, Kaenel relie les débats théoriques aux évolutions politiques d'après la guerre froide et tente d'analyser la résistance européenne aux CS, résistance plus forte en France que dans les pays nordiques. Il passe un peu vite sur la nature et les formes de cette résistance, qui tient, je crois, à la place de l'histoire dans le champ intellectuel français, à l'utilisation différente des théories de la "French theory " mais peut-être aussi à l'influence d'un auteur assez peu cité dans ce recueil, Pierre Bourdieu. Les CS américaines occupent un terrain, intellectuel et universitaire, qui est occupé par la sociologie en France, voire, dans une moindre mesure, par la psychanalyse. La résistance dont il est question n'est pas forcément une peur de la «contamination anglo-américaine " comme Sellier nous le suggère de façon accusatrice, mais vient aussi du fait que certains des outils des CS sont déjà utilisés par d'autres disciplines, c'est leur étiquetage qui diffère. Comme je le suggère plus haut, la visite de librairies ou de bibliothèques universitaires des deux côtés de l'Atlantique est instructive sur ce plan. Là où certains ouvrages seraient classées en critique littéraire, histoire ou sociologie en France, ils peuvent être placés en "African-American interest", CS, Women's studies ou gender studies ou Latino-Latina Studies aux Etats-Unis. Kaenel, comme Sellier, présente la "résistance» aux CS comme un retard européen, la différence est connotée. A la suite de Bourdieu et Wacquant, on pourrait lui reprocher de se faire l'avocat d'un impérialisme disciplinaire qui recoupe un impérialisme culturel américain. Kaenel pourtant, dans de nombreux textes et interventions, déconstruit fort bien l'impérialisme culturel américain et analyse aussi la noncoïncidence entre discours d'ouverture internationale des American Studies et les pratiques qui restent américano-centrées. Article très stimulant donc sur le plan intellectuel qui donne à réfléchir au lien entre politique et recherche universitaire.

13 Le second article d'André Kaenel est d'un grand intérêt également. Intitulé "Moby's Dick and Ahab's Wife: Melville, Gender and Cultural Studies ", il illustre l'impact des CS sur la critique littéraire et le conflit qui existe entre deux approches de la littérature. On aura remarqué la grivoiserie lacanisante du titre qui souligne la prédilection pour le postmodernisme transgressif des CS. Kaenel écrit à la première personne et se cite pour analyser ses propres évolutions face au texte de Melville, dont il est un spécialiste. Son parcours est un exemple de l'impact des CS sur la critique littéraire. En quelque sorte, grâce aux CS, Kaenel introduit des dimensions historiques, sociologiques et féministes dans son approche littéraire. Il est en cela très proche du travail d'Edward Said, sur Jane Austen, par exemple. Cet article risque de ne pas plaire aux tenants d'une critique littéraire qui s'attache à étudier «ce qui fait la spécificité de la littérature » ou «la 
langue » loin de toute « référentialité ». Il ne plaira donc pas à ceux qui pensent qu'il n'y « a pas de hors texte ». Kaenel s'inspire du roman de Sena Jester Naslund, Ahab's Wife, or The Star Gazer, pour relire Moby Dick. On voit ici une application possible des CS, tout au moins des gender studies, à la lecture d'une œuvre canonique. Relecture décentrante et fort intéressante.

Kaenel montre à la fois ce que les CS peuvent apporter à la littérature et pourquoi les critiques derridiens ne peuvent être d'accord avec lui. Son article est une étude de cas et l'une des pistes qu'il ouvre concerne le conflit central entre CS et critique littéraire. En effet, ce n'est pas l'histoire ou la sociologie qui sont le plus affectées par les CS aux Etats-Unis mais la littérature. Les études littéraires américaines varient énormément d'une université à l'autre en fonction de l'impact des CS sur tel ou tel département. C'est dans ce domaine, je crois, que les différences transatlantiques sont les plus importantes et c'est en France que les littéraires sont le plus réticents à adopter les démarches des CS. L'article de Kaenel, théorique et autobiographique, illustre bien ce que l'on pourrait appeler le conflit des disciplines entre critique littéraire et CS.

L'article suivant signé de Michel Imbert est, à mon sens, un article de critique littéraire qui porte sur The American Scene d'Henry James qui, en dépit d'une référence à "la révolution culturelle opérée par les CS » ne fait pas beaucoup appel aux CS. Cette expression de « révolution culturelle » qui renvoie à une sombre période de l'histoire chinoise est d'ailleurs quelque peu problématique. Les CS ont-elles bouleversé le paysage intellectuel ? Le texte d'Imbert est une analyse littéraire classique, d'ailleurs fort différente de celle de Kaenel qui la précède. Là encore se pose le problème du degré de nouveauté des CS. Qu'est ce qui vient de la sociologie, de la linguistique, de l'évolution interne aux disciplines et qu'est-ce qui vient d'une nouvelle méthodologie?

Catherine Bernard clôt le volume par un article portant sur The Politics of Modernism de Raymond Williams. Il s'agit d'un travail d'histoire des idées et de critique littéraire qui est écrit dans une langue bien maîtrisée et souvent philosophique. Article intéressant mais qui, comme le précédent, pose le problème de son inclusion dans un recueil qui étudie surtout l'impact des CS sur tel ou tel champ disciplinaire. Les deux derniers articles ne semblent pas avoir été inclus pour des raisons thématiques et certaines communications du colloque qui n'apparaissent pas ici étaient, à mon sens, plus centrées sur cette thématique.

II.

17 Pourquoi un compte-rendu si long sur un seul ouvrage ? Il faut noter, tout d'abord, la très grande qualité bibliographique de ce recueil. Tous les articles sont suivis d'une bibliographie riche et utile à qui veut poursuivre son apprentissage de l'impact des CS. On voit qu'un soin particulier a été apporté à ces bibliographies qui permettent d'approfondir la réflexion sur les CS. En outre, l'ouvrage balaie bien tout le champ des sciences humaines et sociales (qui comprend l'histoire). Mieux qu'une introduction théorique sur les CS, ce recueil donne les outils et les aperçus nécessaires pour bien saisir les enjeux de l'émergence d'une quasi-discipline (mais aux Etats-Unis seulement). Je voudrais maintenant revenir sur le côté partial et partiel de cet ouvrage qui fait l'apologie des CS en des termes parfois rudes pour les non-adeptes.

18 La critique des CS, de ses évolutions historiques, de ses dérives, passées ou potentielles n'est pas faite. On sait qu'une revue phare de CS, Social Text, a publié, en 1996, un canular sans se rendre compte qu'elle publiait du charabia mais, bien sûr on ne peut incriminer tout un champ disciplinaire sur la base d'une seule dérive. Toute discipline 
s'enrichit et se renforce des critiques internes ou externes. Le débat disciplinaire fait partie de la scientificité. La sociologie de Bourdieu et celle de Boudon n'ont pas grand chose à voir et les débats autour de la définition même de la discipline sont une partie intégrante de la discipline. Gérard Noiriel fait un travail de critique interne à l'histoire fort salutaire. Le dialogue entre Hayden White et Roger Chartier est fort intéressant car s'il est clair que les historiens qui restent positivistes sans le dire et s'imaginent toujours écrire l'histoire wie es gewesen ist, ignorent des problèmes de points de vue, de préjugés personnels ou de corporation que White souligne ; il n'en reste pas moins que Chartier (mais aussi Lynn Hunt, entre autres) a raison d'évoquer le discours de vérité et ce que j'appellerai la résistance du réel.

Il peut sembler étrange que quelqu'un qui a pris la défense de la "civilisation » soit apparemment critique des CS qui, en somme, sont le terme anglo-américain pour désigner le même objet. Néanmoins, il faut noter que dans l'espace culturel angloaméricain les CS n'occupent pas du tout la même place que la «civilisation " puisqu'elles se présentent comme un nouveau paradigme ou même, comme on l'a vu dans ce volume comme une "révolution culturelle». Il y a donc un risque d'hégémonisme disciplinaire qui n'existe aucunement dans un pays comme la France ou la civilisation est une affaire de départements de langues et de concours de recrutement. D'autre part, certaines dérives possibles ont déjà eu lieu.

Le plus grave danger dans les fragmentations disciplinaires américaines qui ont lieu sous l'égide des CS consiste à créer un champ disciplinaire qui correspond à un groupe ethnique ou à un genre. Il est bien entendu tout à fait positif que l'étude des AfricainsAméricains, (comme l'on dit en traduisant mal l'anglais qui place les adjectifs avant les noms là ou le français fait plutôt l'inverse), ou des femmes, aide à faire sortir de l'invisibilité intellectuelle et aide à changer les modes de pensée. Mais, comme le note Guillaume Marche, il y a un risque de communautarisation de la pensée. Si chaque groupe a sa discipline, où sont les possibilités de dialogue commun et où est la scientificité ? La science est-elle condamnée au localisme et se confond-elle avec le militantisme? Il y a une dérive anti-hommes chez certaines féministes américaines comme Andrea Dworkin mais aussi Catharine MacKinnon, dérive qui n'existe pas chez Judith Butler, analyste politique anti-essentialiste hors pair, dérive que l'on ne trouve pas non plus dans les groupes d'étude de la littérature anglo-américaine en France. Il y a une dérive contre-raciste anti-blancs chez certains afrocentristes, dérive qui n'existe pas, loin de là, chez Henry Louis Gates, mais est très présente chez Molefi Kete Asante. Ces dérives font partie du paysage militant habituel et peuvent parfois se comprendre dans un contexte de lutte contre la marginalisation. Néanmoins, si l'université découpe ses disciplines selon des schémas de militantisme ethnique ou sexuel (de genre) la situation devient grave, elle gène le dialogue et la progression de la connaissance qui devient tributaire d'une approbation communautaire. Un spectre hante les CS, celui de l'essentialisme, du repli sur soi, un soi qui n'est plus national mais ethnique ou disciplinaire. Aux « it's a black /woman's /Jewish / gay and lesbian thing/ you wouldn't understand » des uns, favorisés par la fragmentation il faut répondre que l'esclavage, la domination masculine, le génocide, la discrimination sont l'affaire de tous et que la connaissance n'est pas l'affaire d'un groupe spécifique, qu'elle n'est ni génétique ni démographique ni une affaire de « life style enclave».

21 L'article de Jeremy Tranmer permet de mesurer aussi bien le chemin parcouru par les CS depuis leur naissance britannique dans les années 50 que les paradoxes ou ironies 
cocasses dans les retournements des visées des CS. Nées dans le marxisme, les CS en sont venus à se focaliser sur l'ethnicité et le genre en délaissant tous les problèmes de classe. Un des fondateurs britanniques des CS, Richard Hoggart est effaré par le tournant aveugle pris par ce champ. Affirmant étudier "l'inscription sociale des phénomènes culturels ", les CS adoptent souvent les catégories du marketing ou du marché et s'en tiennent à l'injonction lyotardienne de superficialité. Elles suivent en cela le point aveugle de la société américaine et le quasi-consensus sur l'absence de classes sociales. Leurs catégories sont celles de la business culture dont elles n'analysent pas les rapports de pouvoir dans la création culturelle.

Ainsi, alors que les universitaires se mobilisent pour défendre des programmes d'affirmative action qui ont peu d'effets sur l'inclusion des démunis, quelle que soit leur appartenance ethnique, ils acceptent des systèmes universitaires segmentés selon les classes sociales dans lesquels la sélection se fait par l'argent et le capital culturel. En 50 ans et après une traversée transatlantique, les CS ont radicalisé leur vocabulaire mais se sont acculturées au système idéologique dominant qu'elles pensent pouvoir contourner ou transgresser. Il faut rappeler ici, d'ailleurs, que la transgression, comme le carnaval, ne remet pas en cause les systèmes établis, ne les subvertit pas mais les rend tolérables. Thomas Frank, dans son ouvrage One Market Under God, se moque de la dérision des membres de la "cult stud community" vis à vis de l'école de Francfort alors même qu'ils ne font pas l'analyse des industries culturelles et voient des « sites de résistance " dans les talk shows et les magazines de mode. Point aveugle d'une mode universitaire qui n'est pas sans importance. Les talk shows et magazines de mode ou radios rock ne résistent pas, ils célèbrent la consommation y compris par la dérision rituelle et convenue.

La fragmentation disciplinaire qui accompagne une communautarisation de la connaissance épouse le langage de la diversité qui sert de masque idéologique dans une société ou l'inégalité et l'injustice prospèrent. Le paradigme des ethnic studies qui occupe une place considérable dans le champ des sciences humaines gomme des aspects politiques et sociaux importants. Je propose de considérer ce que dit Walter Benn Michaels, professeur à Chicago, par exemple dans un essai publié par le New York Times magazine (11 avril 2004):

What is surprising is that diversity should have become the hallmark of liberalism.

This, if you're on the right, is the gratifying thing about campus radicalism. When student and faculty activists struggle for cultural diversity, they are in large part battling over what skin color the rich kids should have. Diversity, like gout, is a rich people's problem. And it is also a rich people's solution. For as long as we're committed to thinking of difference as something that should be respected, we don't have to worry about it as something that should be eliminated. As long as we think that our best universities are fair if they are appropriately diverse, we don't have to worry that most people can't go to them, while others get to do so because they've had the good luck to be born into relatively wealthy families. In other words, as long as the left continues to worry about diversity, the right won't have to worry about inequality.

Comme la "civilisation» en France, c'est à dire dans nos UFR de langues, les CS proposent une utilisation de divers outils scientifiques adaptés à leur objet. Il ne s'agit donc pas ici de les critiquer en bloc mais de voir comment les affirmations théoriques sont mises en pratique. Le travail d'Edward Saïd, que certains classent dans les CS, d'autres dans la critique littéraire et d'autres encore accusent d'être de la propagande 
politique, me semble novateur. Son objet n'est pas la littérature seule, et il prend soin de distinguer son discours social et politique, de son discours de critique littéraire ou d'amoureux de la littérature. Il peut ainsi apprécier Jane Austen et aussi analyser le contexte socio-économique qui sous-tend son écriture. Son travail ressemble-t-il à celui de certains critiques se revendiquant des CS, qui plaquent une théorie politique sur un texte littéraire, qui lisent à travers un prisme ou des lunettes déformantes qui ne leur laissent voir que leurs propres théories ou préjugés? Par exemple, on ne peut voir en Shakespeare qu'un impérialiste occidental. Le plaquage théorique n'est certes pas l'apanage des CS et l'on voit des lacaniens ne voir dans tous les textes que du Lacan et de la théorie lacanienne tandis que d'autres trouvent du Derrida partout comme d'autres encore avaient placé Marx ou Freud dans toutes leurs analyses. Le plaquage théorique est d'ailleurs plus fréquent en France ou « le démon de la théorie » dont parle Antoine Compagnon semble plus vif qu'ailleurs. Les textes alors ne deviennent que des illustrations d'une théorie pré-existante et toute puissante. Les CS favorisent l'intervention des « interpreting communities " (Stanley Fish) communautaristes dans la connaissance et donc le plaquage d'une doctrine pré-établie sur les textes et l'analyse des institutions.

Le recueil propose une traduction en français des CS, traduction que les auteurs, mêmes les directeurs et le coordonateur ne reprennent pas. Tzvetan Todorov n'hésite pas à dire qu'il pratique maintenant les «études culturelles ». Il utilise ses connaissances en sciences humaines et les divers outils qui lui semblent appropriés pour telle ou telle étude. Il fait ce que tout bon civilisationniste se doit de faire. Il s'agit alors d'un classement fonctionnel et d'une méthode, pour une pratique aux origines scientifiques diverses. Pourquoi la traduction française de CS ne s'est-elle pas imposée ? Cette résistance linguistique ne peut venir d'un refus de "contamination", au contraire, puisque le terme anglais a droit de cité et que l'origine des CS est en partie française. Qu'est-ce qui empêche la "science » des CS d'être universelle comme la sociologie ou l'histoire? Certes, les disciplines des sciences humaines, comme nous l'avons vu, sont moins perméables à une interrogation multiforme mais aussi, je crois, elles reconnaissent dans les CS des outils émanant de disciplines répertoriées et refusent le localisme de la science. Les CS américaines sont souvent trop ancrées sur un groupe pour bien s'exporter en dehors du cadre des études américaines.

En Europe, le souvenir des dérives du stalinisme dans les sciences a laissé des traces, la biologie à la Lyssenko ou la création et la critique littéraire du "réalisme socialiste » ont laissé de mauvais souvenirs. L'idée que la connaissance puisse être l'apanage, la possession ou la prérogative d'un groupe, qu'il soit le prolétariat d'antan ou tel ou tel groupe ethnique ou culturel aujourd'hui, passe mal. La sociologie de Bourdieu est à la fois ancrée dans un champ intellectuel français mais aussi internationale et surtout internationalisable. Le multiculturalisme de discours aux Etats-Unis s'accommode bien d'une ségrégation revendiquée à l'université, où non seulement les dortoirs mais aussi les activités culturelles sont séparés au nom d'une idéologie de la tolérance et de la diversité. Les annonceurs ont accepté la segmentation de leurs marchés selon les normes du nouveau séparatisme culturel et les universitaires entérinent eux aussi la segmentation de leurs objets de recherche et d'enseignement. Ainsi des sous-ensembles disciplinaires des CS, comme les Women's Studies ou les African-American studies qui avaient pour but initial de rendre visibles les hommes et les femmes invisibilisés par la culture dominante ont débouché, parfois, vers une fermeture contre-raciste ou contre- 
sexiste, mais toujours essentialiste à rebours, qui exclut les membres extérieurs à ces groupes des cours de ces départements ou programs. (ce qui ne se passe pas en France, pays « retardataire », resté universaliste, ou jacobin, selon certains) Les CS participent de ce nouveau ségrégationnisme inversé et au système des « life style enclaves » mis au point par les publicitaires ; elles atteignent alors un but strictement opposé à celui des premiers praticiens britanniques. Dans son livre le plus récent, parfois assez contradictoire, Terry Eagleton déclare «Culture had been among other things a way of keeping radical politics warm, a continuation of it by other means. Increasingly, it was to become a substitute for it. In some ways, the 1980s were like the 1880 s or 1960 s without the politics » (p. 45). Il s'agit là d'une idée que T. Gitlin avait déjà développée aux Etats-Unis où les «radicals » ont pris le département d'anglais tandis que la droit prenait la Maison Blanche (The Twilight of Common Dreams). Les CS apparaissent alors comme un ersatz de politique, que certains universitaires «traditionalistes ", (comme John Ellis, par exemple), regrettent, avec des argumentaires que l'on retrouve souvent chez nos collègues littéraires français, mais cette pseudo-politique n'est pas pour déplaire à la droite politique. Une analyse de la fonction politique et sociale des CS aurait donc été la bienvenue dans cet ouvrage.

Ainsi un ouvrage excellent qui présente les CS et leurs apports ou impacts sur divers champs disciplinaires se montre très partisan et partial. Il n'analyse pas les apories des CS ni les décalages entre théories et pratiques, ni les décalages entre affirmations de nouveauté radicale et la filiation avec des disciplines plus anciennes. Il n'aborde que très peu les problèmes de définition et les rapports entre post-modernisme, déconstruction, theory ou French theory et CS. Je me souviens avoir lu, dans les années 80 , un échange entre le psychanalyste Octave Mannoni et le sociologue Robert Castel dans lequel chacun rappelait à l'autre que sa discipline avait quelques contradictions et difficultés à prendre en compte. Il en est de même pour chaque discipline d'ailleurs. L'histoire de Chartier et celle de Kaspi sont elles les mêmes disciplines? La psychanalyse de Lacan et celle de Winnicott ou de Freud? La critique littéraire de Derrida et celle de Bloom (Harold ou Allan) ? Les affaires Goldhagen, en histoire, ou Tessier, en sociologie, montrent qu'il n'y a pas consensus sur la discipline à l'intérieur d'elle-même. Il y a donc débat à l'intérieur des CS, débat sur la définition, débat sur les pratiques, débat à l'extérieur des CS et la présentation des CS aurait dû inclure des références à ces débats, inclure une analyse des limites et impasses des CS et interroger ce schéma implicite dans de nombreux articles selon lequel les «CS angloaméricaines » sont le modèle à suivre que seuls des retardataires se refusent à faire. De façon cocasse d'ailleurs, Eagleton annonce, dans le monde anglophone, le dépassement de ce modèle largement inspiré de penseurs français, que certains ici nous encouragent à imiter.

Si l'ouvrage fait une place importante et justifiée aux origines britanniques des CS, il n'analyse pas l'apport français et surtout les transformations induites par le passage d'une culture nationale et/ou universitaire à une autre. Derrida passe de France (ou plutôt du français) aux Etats-Unis mais aussi de la philosophie aux études littéraires. Son travail diffère sensiblement de celui de Foucault ou de Baudrillard (qui aurait aimé faire oublier Foucault). Le champ des CS a créé une fausse impression de cohérence entre penseurs dont le seul lien commun était l'origine nationale et la langue. Il s'agit ici d'éviter un étiquetage national ou nationaliste mais aussi d'analyser le transfert des idées d'une culture à l'autre. Les clivages, d'ailleurs, sont plus intellectuels, idéologiques et de cultures universitaires que nationaux, comme l'a montré l'affaire 
Sokal. On pourra néanmoins lire avec intérêt l'ouvrage de François Cusset, French Theory, Foucault, Derrida, Deleuze et Cie et les mutations de la vie intellectuelle aux Etats-Unis, qui fait le point sur ces auteurs, selon une démarche quelque peu marketing de la pensée française.

Aux Etats-Unis c'est surtout dans le champ littéraire que les CS font rage. Les historiens reprochent aux CS de ne pas faire de travail d'archives, les sociologues et les anthropologues de ne pas faire de terrain. L'apport des CS dans ces sciences sociales est un apport d'analyse de discours informé par la critique littéraire et la psychanalyse. En littérature, les CS apportent un discours politique ou social là où il n'y avait qu'un discours textuel. En somme, les CS font dialoguer les disciplines et les résultats sont parfois excellents. Dans d'autres cas, un champ disciplinaire est réduit à l'apport de l'analyse de discours.

30 Ainsi, on peut introduire de la psychanalyse pour analyser le discours machiste d'un Theodore Roosevelt et ainsi apporter une dimension supplémentaire à l'analyse historique mais cet angle d'attaque ne peut être le tout d'une analyse. Il me semble souvent, à côtoyer des spécialistes de politique étrangère ou des politologues, que leurs analyses bénéficieraient d'un contact avec la sociologie, la critique littéraire ou la psychanalyse car souvent ils sont pris dans un réseau de préjugés ou croyances nonanalysées qui gène leur appréhension de leur propre objet. (Je laisse de côté les économistes tant le bain idéologique dans lequel ils trempent a déjà été souvent analysé). Mais ce côtoiement ne saurait remplacer tout le travail proprement politique ou historique. Il y a ici un aspect des CS qui n'est pas nouveau du tout. En effet, écrivains et sociologues, écrivains et politologues ont toujours eu des débats et controverses sur les mêmes objets et la littérature a souvent devancé la politique, parfois les politistes ont corrigé les ardeurs des écrivains, par exemple la désinvolture pro-chinoise d'un Sollers qui, jeune vedette de Tel Quel, jouait avec la révolution culturelle.

31 Les concepteurs de ce recueil ont choisi de "positiver " (accentuate the positive) et de ne présenter que la face solaire des CS. Face indéniable mais il faut analyser les gains tout autant que les pertes, Edward Said, qui n'est pas vraiment postmoderne, tout autant que les ânes qui ont publié le canular de Sokal, ne faisant pas la différence entre jargon abscons et CS. Alors que la plupart des articles de cet ouvrage sont excellents, notamment ceux des concepteurs, on ne peut s'empêcher de penser que dans la conception de leur recueil, ils ont eux aussi, comme leur objet, succombé à la logique publicitaire. Logique publicitaire, puisque seuls les mérites sont vantés. Le tour d'horizon de ces universitaires aurait dû être plus critique. Le tout d'un ouvrage doit être plus que la somme de ses parties. Ici les parties sont de très bonne qualité mais le tout laisse à désirer, (les lacaniens s'en réjouiront).

On aura compris, grâce, entre autres, à la longueur de ce compte-rendu, que cet ouvrage est très stimulant et important et que l'on ne peut qu'en recommander la lecture à tous les civilisationnistes ou amateurs de théorie en critique littéraire. Néanmoins, il ne correspond pas à son titre car il laisse de côté plusieurs facettes importantes du phénomène complexe des CS et notamment sa non traduction en France et son flirt méthodologique avec l'industrie du marketing et de la communication. Assez étrangement, cet ouvrage est un peu en retard sur les évolutions aux Etats-Unis où le système de la mode est en train de périmer de nombreux aspects 
flamboyants des CS. Mais ne pas être à la pointe de la mode n'a pas que des inconvénients.

INDEX

Thèmes : Recensions

AUTEUR

PIERRE GUERLAIN

Université Nanterre-Paris 10 